\title{
Clinicopathological Features and Prognosis of Gastrointestinal Stromal Tumors: A Study on 250 Cases
}

\author{
Ya-Hong Li ${ }^{1}$, Hong-Xia Xie ${ }^{2}$, Yong-Hong Shi ${ }^{2}$ Zhao-Xia Cui ${ }^{3}$, and Ming Liu²,* \\ ${ }^{1}$ Inner Mongolia Medical University, Inner Mongolia, China \\ ${ }^{2}$ The Affiliated Hospital of Inner Mongolia Medical University, Inner Mongolia, China \\ 3Junshi Biosciences, Shanghai, China
}

*Corresponding author: Ming Liu, The Affiliated Hospital of Inner Mongolia Medical University, Inner Mongolia, China, E-mail: liumingfy@163.com

Received: 24 Oct, 2019 | Accepted: 08 Nov, 2019 | Published: 15 Nov, 2019

Citation: Ya-Hong L, Hong-Xia X, Yong-Hong S, Zhao-Xia C, Liu M (2019) Clinicopathological Features and Prognosis of Gastrointestinal Stromal Tumors: A Study on 250 Cases. Clin Res Open Access 5(2): dx.doi.org/10.16966/2469-6714.151

Copyright: (c) 2019 Ya-Hong L, et al. This is an open-access article distributed under the terms of the Creative Commons Attribution License, which permits unrestricted use, distribution, and reproduction in any medium, provided the original author and source are credited.

\begin{abstract}
The clinicopathological data, immunohistochemistry and partial genotyping of 250 patients with primary GIST treated in the Affiliated Hospital of Inner Mongolia Medical University from January 2013 to January 2018 were retrospectively analyzed. The results demonstrated that among the 250 patients in our hospital, 131 were male and 119 were female, aged from 20 to 86 years, with a median age of 60 years. It mainly occurred in the stomach (58.4\%), and in the small intestine (24.0\%). 171 cases of patients with mitotic count $<5 / 50 \mathrm{HPF}$, and the rest of patients with mitotic count $>5 / 50$ HPF. 15 cases of patients with tumor diameter less than $2 \mathrm{~cm}, 88$ cases of patients with 2-5 cm, $110 \mathrm{cases}$ with $5.1-10 \mathrm{~cm}$, and the others with tumor diameter larger than $10 \mathrm{~cm}$. According to modified NIH criteria, the risk was divided into: 15 very low risk patients, 66 low risks, 68 intermediate risks and 110 high risk. The positive rates of immunohistochemical markers CD117, DOG-1 and CD34 were $96.4 \%, 90.4 \%$ and $77.2 \%$ respectively. There were significant differences in the expression of CD117 and DOG-1 in different tumor sizes and modified NIH criteria (P<0.05), and the expression of CD34 in different mitotic count $(P<0.05)$. Among the 250 patients, 16 patients were treated with imatinib because of the large size and location of the tumors. 17 patients with metastasis mainly metastasized to the liver, the primary site was the stomach, and most of the metastasis patients had mutations in exon 11 of c-kit. The prognosis of small intestine and rectum after operation is obviously worse than those of stomach. The overall prognosis of this group is good and the results can objectively reflect the current situation of diagnosis and treatment of GIST in our hospital.
\end{abstract}

Keywords: Gastrointestinal stromal tumor; Retrospective analysis; Imatinib; Prognosis; Immunohistochemistry

\section{Introduction}

Gastrointestinal Stromal Tumors (GIST) are the most common mesenchymal tumors in the gastrointestinal tract [1], accounting for $1-3 \%$ of all malignant gastrointestinal tumors and $5-7 \%$ of sarcomas [2]. Most of them occur among middle-aged and elderly patients and the incidence is lower among children and young people [3,4], sometimes related to family or heredity [5,6]. Most studies reported that the annual incidence of GIST was 10-15/million, with no significant geographical difference [7]. The most common sites were stomach $(60 \%)$ and jejunum/ileum $(30 \%)$ and less in duodenum, colon, rectum, appendix and esophagus $(<15 \%)$. Gastrointestinal stromal tumors (GIST) have no specificity, and the course of disease can be from benign to malignant. In earlier stage, they are usually asymptomatic or mild. Later clinical symptoms depend on the size and location of the tumors, which mainly include anaemia due to occult bleeding, nausea, vomiting, abdominal pain, abdominal mass, abdominal distention, obstructive jaundice, constipation or diarrhea, frequent urination, and fatigue [8]. GIST of the stomach tend to be benign, where they outnumber malignant GIST by a ratio of 3-5:1 [9]. Computed Tomography (CT) is the most commonly used method for initial evaluation and staging of GIST. It can determine the location, size and metastasis of tumors, and guide biopsy for diagnosis. GIST has low specificity and can be diagnosed according to morphology (spindle cells, epithelioid cells or mixed cells) and Immunohistochemistry (IHC) of CD117, DOG-1 and CD34. GIST metastases are most common in the liver (28\%), mesentery and omentum (30\%), but less commonly in the lung (7\%), subcutaneous tissue (4.7\%), lymph nodes (4.7\%) or bone metastasis (2.3\%) [10].

Surgery is the main method for the treatment of GIST. According to NCCN and ESMO guidelines, the treatment of incidental small GIST less than $2 \mathrm{~cm}$ has been controversial [11], while complete tumor excision is mandatory for GIST with size $>2 \mathrm{~cm}$, with the laparoscopic wedge resection to be a feasible and safe approach [12]. For patients with larger tumors, laparoscopic surgery is controversial and traditionally open surgery should be applied. The pathogenesis of GIST is related to the genetic mutation of kit and PDGFRA. Imatinib, Sunitinib and Regorafenib are Tyrosine Kinase Inhibitors (TKI) $[13,14]$. For patients with larger tumors, imatinib can be administered orally for 6-12 months, and surgical treatment can be performed after the tumor shrinks, which can preserve the function of important 
organs and increase the chance of radical resection. The risk of recurrence and metastasis of high-risk GIST is very high, and orally administered imatinib for 1-3 years after operation can effectively reduce the risk of recurrence and metastasis.

\section{Materials and Methods}

\section{Clinical data}

A retrospective analysis was made of the clinicopathological data of 308 patients with GIST treated in the Affiliated Hospital of Inner Mongolia Medical University from January 2013 to January 2018. Among them, 23(7.4\%) were malignant tumors of digestive tract accompanied by GIST, 15(4.9\%) patients had incomplete data, $20(6.5 \%)$ patients failed to follow up. The remaining 250(81.2\%) were primary GIST and were analyzed for the correlation between CD117, DOG-1, CD34 and different clinicopathological characteristics (age, gender, tumor site, tumor size, mitotic index, modified Nation Institute of Health (NIH). Sixteen patients with large tumors were treated with imatinib before and after operation. The changes of tumor size and pathological immunohistochemistry before and after treatment were analyzed. The relationship between recurrence and metastasis and the original site was analyzed in 17 patients. The latest follow-up was in December 2018

\section{Statistical methods}

2008 modified NIH criteria was used to grade the tumors according to tumor size, mitotic index, tumor location and whether the tumor ruptured or not. Follow-up was conducted by telephone inquiry, outpatient or inpatient review. Survival time was from the time of diagnosis to the date of death or last follow-up. SPSS20.0 statistical software was used for statistical analysis of the collected data, and the counting data were expressed by relative number and $\mathrm{X}^{2}$ test. The measurement data were expressed by $\mathrm{X} \pm \mathrm{S}$ and $\mathrm{T}$ test. And the survival analysis was carried out by Kaplan-Merie. $\mathrm{P}<0.05$ was statistically significant.

\section{Results}

\section{Correlation between immunohistochemistry and clinicopathological characteristics}

In the diagnosis and treatment of the 250 patients in our hospital, there were 131 males and 119 females, with a male-to-female ratio of $1.1001: 1$, and there was no significant difference. The median age was 60 years (20-86), 3 patients aged 20-30 years, 10 patients aged 31-40, 40 patients aged $41-50$, and 82 patients aged $51-60,83$ patients aged $61-70,30$ patients aged $70-80,2$ patients aged over 80 , and the high incidence age of GIST was 51-70 years. GIST mainly occurred in the stomach with 146 cases (58.4\%), followed by small intestine of 60 cases (24\%), duodenum 13 cases, colon 4 cases and other 17 cases. Mitotic count of 171 cases was less than 5/50 HPF, and that of other patients was more than 5/50 HPF. There were 15 patients with tumor diameter less than $2 \mathrm{~cm}, 88$ patients with tumor diameter of $2-5 \mathrm{~cm}, 110$ patients with tumor diameter of 5.1-10 cm, and the rest patients with tumor diameter greater than $10 \mathrm{~cm}$. According to $\mathrm{NIH}$ criteria, the risk was divided into the following: 15 very low risk patients, 66 low risks, 68 intermediate risks and 110 high risk.

The positive rates of CD117, DOG-1 and CD34 were 96.4\% (241), $90.4 \%$ (226) and $77.2 \%$ (193) respectively. There were 190 cases of double positive expression of CD34 and CD117 protein, 180 cases of double positive expression of CD34 and DOG-1 protein; and there were significant differences between CD34 and CD117 and DOG1 protein in 250 cases of GIST $(\mathrm{P}<0.05)$. The expression of CD117 and DOG-1 in different tumor sizes and modified NIH criteria had statistical significance $(\mathrm{P}<0.05), \mathrm{CD} 34$ expression in different mitotic index had statistical significance $(\mathrm{P}<0.05)$, and $\mathrm{CD} 34$ positive rate was higher when mitotic count $>5 / 50 \mathrm{HPF}$ (Table 1 ).

\section{Preoperative treatment of imatinib}

The National Comprehensive Cancer Network (NCCN) guidelines recommend imaging monitoring of response to imatinib therapy. Among the 250 patients treated in our hospital, 16 patients with larger tumors received imatinib for 6-9 months before surgery. The positive expression rate of CD117 was significantly reduced (Figure 1). CT scans were performed every three months. Table 2 shows that the diameter and CT value of tumors tended to decrease. There was significant difference in tumor size among groups $(\mathrm{P}<0.05)$. The risk of operation was reduced and radical resection was performed. The patients survived well after operation.

\section{The correlation between the primary site and metastasis site of GIST}

Among the 250 cases, 17 (6.8\%) had metastasis; the age range for metastatic patients is 42 to 79 years old. Most of the metastases were to the liver (4.8\%) and were mainly from the stomach and small intestine; while most of the duodenum metastasized to the abdominal cavity. The relationship between primary location and metastatic location is shown in table 3. The mutation rate of c-kit was $70.59 \%(12 / 17)$, followed by exon 9 mutation in exon 11, PDGFR mutation rate was 23.53(4/17), mainly exon 18 mutation, and one wild-type mutation. Early treatment of patients with GIST is critical. In 17 patients with recurrence and metastasis, 3 died within five years.

\section{Prognosis of different location of GIST}

GIST can occur in any part of the digestive tract from esophagus to rectum. Survival curves were drawn by Kaplan-Meier to analyze the prognosis of different locations of the patients in our hospital. Figure 2 shows that the overall effect of the patients after operation is better, and the prognosis of stomach and colon is better than that of small intestine and rectum.

\section{Discussion}

Gastrointestinal stromal tumors are mesenchymal gastrointestinal tumors with diverse clinical manifestations. The degree of invasion varies with the location, size and mitotic index [15]. The median age of the patients in our study was 60 years, the youngest was 20 years, and the oldest was 86 years. Among the 36 cases of GIST studied by Alghamdi HM, et al. [16] in Saudi Arabia, the range of cases was 17-81 years, with no difference in the age of onset. Among the 250 patients with GIST in our study, $58.4 \%$ were originally located in the stomach, followed by $24 \%$ in the small intestine. In the Boston Fairweather, et al. [17] study, 323 patients, $41 \%$ were in the stomach and $43 \%$ in the small intestine. In the study of Liu Xiaowei, et al. [18] in southern China, $45.08 \%$ were in the stomach and $23.77 \%$ in the small intestine. In our study, the gastric incidence was slightly higher than that of others, and the incidence of small intestine in northern and southern China was the same, which was slightly lower than the incidence of small intestine GIST in Boston.

CD34 can significantly express in vascular endothelial cells and hematopoietic precursor cells, promoting angiogenesis, so diffuse expression of CD34 can promote tumor growth and lead to tumor metastasis [19]. CD117 is a C-kit receptor whose activity can activate downstream signal transduction pathway for a long time, hinder the normal proliferation and apoptosis of cells, and ultimately 
Table 1: The expression of immunohistochemistry in different clinical features.

\begin{tabular}{|c|c|c|c|c|c|c|c|}
\hline \multirow[b]{2}{*}{ Clinicopathologic characteristics } & \multirow[b]{2}{*}{ Patients $(n=250)$} & \multicolumn{2}{|c|}{ CD117 } & \multicolumn{2}{|c|}{ DOG-1 } & \multicolumn{2}{|c|}{ CD34 } \\
\hline & & $\begin{array}{c}+ \\
(n=241)\end{array}$ & $\mathbf{P}$ & $\begin{array}{c}+ \\
(n=226)\end{array}$ & $\mathbf{P}$ & $\begin{array}{c}+ \\
(n=193)\end{array}$ & $\mathbf{P}$ \\
\hline Gender & & & 0.626 & & 0.498 & & 0.064 \\
\hline Male & 131 & 127 & & 120 & & 95 & \\
\hline Female & 119 & 114 & & 106 & & 98 & \\
\hline Age (yr) & & & 0.487 & & 0.595 & & 0.711 \\
\hline $20-30$ & 3 & 3 & & 3 & & 2 & \\
\hline $31-40$ & 10 & 9 & & 8 & & 6 & \\
\hline $41-50$ & 40 & 40 & & 34 & & 31 & \\
\hline $51-60$ & 82 & 77 & & 74 & & 67 & \\
\hline $61-70$ & 83 & 80 & & 76 & & 63 & \\
\hline $71-80$ & 30 & 30 & & 29 & & 23 & \\
\hline$>80$ & 2 & 2 & & 2 & & 1 & \\
\hline Tumor location & & & 0.594 & & 0.737 & & 0.437 \\
\hline Gastric & 146 & 139 & & 133 & & 118 & \\
\hline Duodenum & 13 & 13 & & 12 & & 10 & \\
\hline Small intestine & 60 & 59 & & 54 & & 44 & \\
\hline Rectum & 10 & 9 & & 8 & & 8 & \\
\hline Colon & 4 & 4 & & 3 & & 2 & \\
\hline Other & 17 & 17 & & 16 & & 11 & \\
\hline Mitotic index & & & 0.178 & & 0.464 & & 0.009 \\
\hline Less than $5 / 50 \mathrm{HPF}$ & 171 & 163 & & 153 & & 124 & \\
\hline$>5 / 50 \mathrm{HPF}$ & 79 & 78 & & 73 & & 69 & \\
\hline Tumor size & & & 0.00 & & 0.002 & & 0.124 \\
\hline Less than 2 & 15 & 12 & & 10 & & 8 & \\
\hline $2-5$ & 88 & 82 & & 76 & & 69 & \\
\hline $5-10$ & 110 & 110 & & 105 & & 91 & \\
\hline$>10$ & 37 & 37 & & 35 & & 25 & \\
\hline Modified NIH & & & 0.003 & & 0.003 & & 0.067 \\
\hline Very long risk & 15 & 12 & & 10 & & 8 & \\
\hline Low risk & 66 & 64 & & 59 & & 48 & \\
\hline Intermediate risk & 68 & 65 & & 60 & & 56 & \\
\hline High risk & 101 & 100 & & 97 & & 81 & \\
\hline
\end{tabular}

Table 2: Tumor size before and after imatinib treatment of changes.

\begin{tabular}{|l|c|c|c|}
\hline \multicolumn{1}{|c|}{ Project } & Before treatment & $\mathbf{6}$ months & 9 months \\
\hline Diameter $(\mathrm{mm})$ & $95.7 \pm 34.5$ & $58.1 \pm 33.4$ & $42.2 \pm 27.3$ \\
\hline CT value $(\mathrm{Hu})$ & $73.6 \pm 13.2$ & $44.8+14.3$ & $39.8+12.5$ \\
\hline
\end{tabular}

lead to tumorigenesis [20]. A chloride channel protein encoded by DOG- 1 expressed in cell membrane and CD117 and DOG-1 are the pathological diagnosis recommended by NCCN and the consensus of diagnosis of gastrointestinal stromal tumors in China. The positive rates of CD117, DOG-1 and CD34 in 250 patients were $96.4 \%, 90.4 \%$ and $77.2 \%$ respectively. The expressions of CD117 and DOG-1 were similar to those reported in previous literatures [21]. The expression of CD34 was slightly higher than that of other studies [22]. Immunohistochemistry is indispensable for the final diagnosis of GIST, in which CD117, DOG-1 and CD34 are commonly used marker proteins. Immunohistochemical detection after tumor surgery can not only confirm the diagnosis, but also provide basis for further detection of mutations in C-kit and PDGFRa gene hotspots and provide a direction for postoperative molecular targeted therapy.
Table 3: The relationship between primary site and metastasis site.

\begin{tabular}{|l|c|c|c|c|}
\hline \multirow{2}{*}{$\begin{array}{c}\text { Primary location } \\
\text { of GIST }\end{array}$} & Liver & Abdominal cavity & Lymphoma & Total \\
\cline { 2 - 5 } & 4 & 0 & 1 & 5 \\
\hline Stomach & 2 & 2 & 0 & 4 \\
\hline Duodenum & 4 & 0 & 2 & 6 \\
\hline Small intestine & 2 & 0 & 0 & 2 \\
\hline Other & 12 & 2 & 3 & 17 \\
\hline Total & & & & \\
\hline
\end{tabular}

Tumor metastasis is a multi-step and multi-link process, including spontaneous exfoliation of tumor cells, infiltration of surrounding tissues into the circulatory system. To break through the formation of capillary metastases, in which neovascularization is an important link in the invasion and metastasis of tumors. At our hospital from 2013-2018, 17 cases (6.8\%) in the diagnosis and treatment of patients with metastatic, mainly to the liver (4.8\%), and primary site is mainly at the stomach $(41.18 \%)$. Liver metastatic rate is higher than that of Wang's research, 825 cases of GIST patients, 45 cases (5.45\%) of the 

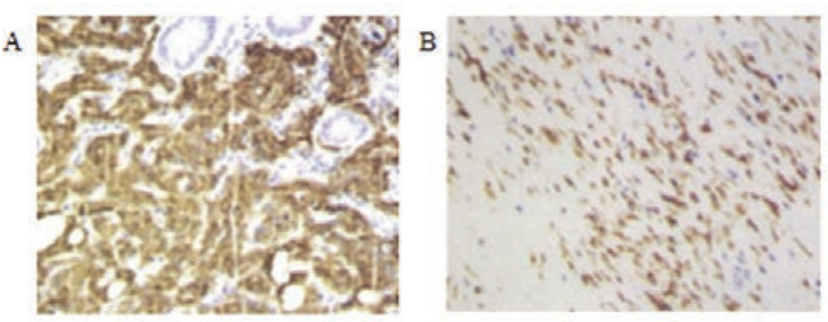

Figure 1: CD117 changes before and after treatment of imatinib (typical cases)

A-Before imatinib treatment, HE staining; magnification, $10 \times 20$

B-After imatinib treatment, HE staining; magnification, $10 \times 20$

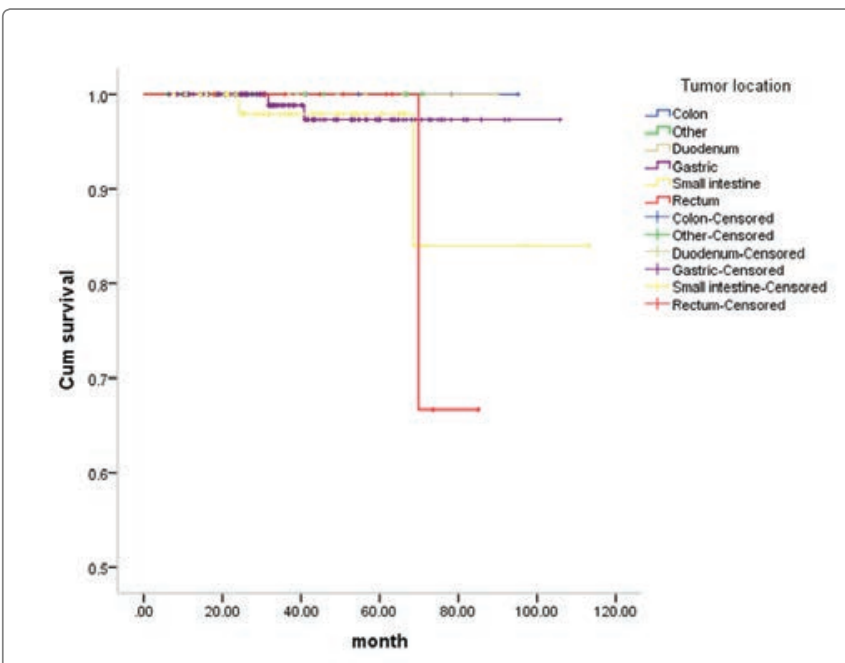

Figure 2: Survival curve at the site of the disease.

metastatic, including liver metastasis occurred in 11 cases (1.33\%), lymph node metastasis in $9(1.09 \%)$, peritoneal metastasis occurred, 25 cases $(2.91 \%)$. The youngest patients with metastatic tumor were 23 years old, and the oldest was 82 years old. The most common age is 50-60 years old. The most common primary site was the stomach in 24 cases (53\%), followed by the small intestine in 15 cases (30\%) [23]. In Li's research [24], c-Kit exon 11 mutations in high risk GIST prone to secondary mutations can lead to loss of exon 11 mutation tended to cause post-operation relapse and metastasis. This can be used as one of the independent factors of poor prognosis, metastasis patients. In the study of this group most Kit mutation was in exon 11. Types of gene mutation detection of GIST are of great significance to implement individualized accurate treatment and prognostic assessment according to the characteristics of genetic mutations in patients with GIST. The high recurrence rates might be caused by the multifocal nature of this disease process; the identification of additional tumor foci with initial imaging studies provides valuable information for the surgeon.

GIST can occur in any part of the gastrointestinal tract and abdominal cavity, and the prognosis is different in different parts. The prognosis of the stomach is significantly better than that of the small intestine and rectum, which is consistent with the study of Chou FF, et al. [25]. The poor prognosis may be manifested in recurrence, distant metastasis and invasion of adjacent tissues. At present, complete surgical resection of the lesion is an effective method for the treatment of metastatic tumor of GIST. For patients with genetic mutations, imatinib treatment before surgery can reduce the tumor size, reduce surgical risk, narrow the surgical scope, and improve the surgical resection rate of the tumor that is difficult to be removed. At the same time, it can be used as postoperative adjuvant therapy, which can significantly reduce the recurrence rate of tumors. Postoperative adjuvant therapy for GIST patients with high risk of recurrence should be based on 3-year adjuvant therapy [26].

GIST is characterized by co-expression of CD117, DOG-1 and $\mathrm{CD} 34$, and its important molecular mechanism is acquired mutation of c-kit and PDGFR a genes. At present, it is believed that the main factors related to the prognosis of GIST are modified NIH criteria, tumor diameter, mitotic index, and the integrity of surgical resection [27]. Imatinib has good clinical effects in patients with unresectable and metastatic GIST.

\section{Conclusion}

To sum up, the detection of immunohistochemical markers CD117, DOG-1 and CD34 is of great significance in the diagnosis of gastrointestinal stromal tumors. Imatinib has certain significance in the preoperative treatment of patients with large tumors and is also a long-term drug for patients with metastasis. Gastric and small intestine tumors mainly metastasis to the liver; while the prognosis of small intestine is poor. Middle-aged and elderly people are the highrisk population of GIST, and the main sites of the disease are stomach and small intestine. The overall prognosis of this group is good. The results of this study can objectively reflect the current situation of diagnosis and treatment of GIST in our hospital.

\section{References}

1. Cioffi A, Maki RG (2015) GI Stromal Tumors: 15 years of lessons from a rare cancer. J Clin Oncol 33: 1849-1854.

2. DeMatteo RP, Lewis JJ, Leung D, Mudan SS, Woodruff JM, et al. (2000) Two hundred gastrointestinal stromal tumors; recurrence pattern and prognostic factors for survival. Ann Surg 231: 51-58.

3. Kim HC, Lee JM, Kim KW, Park SH, Kim SH, et al. (2004) Gastrointestinal stromal tumors of the stomach: $\mathrm{CT}$ findings and prediction of malignancy. AJR Am J Roentgenol 183: 893-898.

4. Burkill GJ, Badran M, Al-Muderis O, Meirion Thomas J, Judson IR, et al. (2003) Malignant gastrointestinal stromal tumor: distribution, imaging features, and pattern of metastatic spread. Radiology 226 : 527-532.

5. Marrari A, Wagner AJ, Hornick JL (2012) Predictors of response to targeted therapies for gastrointestinal stromal tumors. Arch Pathol Lab Med 136: 483-489.

6. Joensuu H, Hohenberger P, Corless CL (2013) Gastrointestinal stromal tumour. Lancet 382: 973-983.

7. Søreide K, Sandvik OM, Søreide JA, Giljaca V, Jureckova A, et al. (2016) Global epidemiology of gastrointestinal stromal tumours (GIST): A systematic review of population-based cohort studies. Cancer Epidemiol 40: 39-46.

8. Dagher R, Cohen M, Williams G, Rothmann M, Gobburu J, et al. (2002) Approval summary: Imatinib mesylate in the treatment of metastatic and/or unresectable malignant gastrointestinal stromal tumours. Clin Cancer Res 8: 3034-3038.

9. Sandrasegaran K, Rajesh A, Rydberg J, Rushing DA, Akisik FM, et al. (2005) Gastrointestinal stromal tumors: clinical, radiologic, and pathologic features. AJR Am J Roentgenol 184: 803-811. 
10. Sujata Patnaik, Yarlagadda Jyotsnarani, Susarla Rammurti (2012) Radiological features of metastatic gastrointestinal stromal tumors. J Clin Imaging Sci 2: 43.

11. Wysocki WM, et al. (2015) Postpowanie w przypadku sporadycznej w kniakowatoci typu desmoidu (stanowisko EORTC I SPAEN Z 2014 r.) Med Prakt Onkol 3: VII-IX.

12. Casali PG, Abecassis N, Aro HT, Bauer S, Biagini R, et al. (2018) Gastrointestinal stromal tumours: ESMO-EURACAN clinical practice guidelines for diagnosis, treatment and follow-up. Ann Oncol 29: iv68-iv78.

13. Buchdunger E, Zimmermann J, Mett $\mathrm{H}$, Meyer T, Müller $\mathrm{M}$, et al. (1996) Inhibition of the Abl protein-tyrosine kinase in vitro and in vivo by a 2-phenylaminopyrimidine derivative. Cancer Res 56: 100 104.

14. Demetri GD, Reichardt P, Kang YK, Blay JY, Rutkowski P, et al. (2013) Efficacy and safety of regorafenib for advanced gastrointestinal stromal tumours after failure of imatinib and sunitinib (GRID): an international, multicentre, randomised, placebo-controlled, phase 3 trial. Lancet 381: 295-302.

15. Miettinen M, Lasota J (2006) Gastrointestinal stromal tumors: pathology and prognosis at different sites. Semin Diagn Pathol 23: 70-83.

16. Alghamdi HM, Amr SS, Shawarby MA, Sheikh SS, Alsayyah AA, et al. (2019) Gastrointestinal stromal tumors A clinicopathological study. Saudi Med J 40: 126-130.

17. Fairweather M, Balachandran VP, Li GZ, Bertagnolli MM, Antonescu C, et al. (2018) Cytoreductive Surgery for Metastatic Gastrointestinal Stromal Tumors Treated with Tyrosine Kinase Inhibitors: A 2-institutional Analysis. Ann Surg 268: 296-302.

18. Liu Xiaowei, Liu Rensheng, Zheng Qichang (2018) Diagnosis, Treatment and Prognosis of 122 Cases of Gastrointestinal Stromal Tumors. Medical Journal of Wuhan University 39: 660-662.
19. Li Wangyong, Chen Guofu, Yang Xiaoyan, Han Shaoliang, Wu Xiuling (2015) Expression and clinical significance of CD133 and CD44 in gastrointestinal stromal tumors tissues. Journal of Chinese Physician 17: 1698-1701.

20. Niu $Q$, et al. (2015) The clinical feature, pathological characterstics and treatment of 174 cases of gastricintestinal stromal tumor. Chin J Gastroentarol Hepatol 24: 728-730.

21. Blackstein ME, Blay JY, Corless C, Driman DK, Riddell R, et al. (2006) Gastrointestinal stromal tumors: consensus statement on diagnosis and treatment. Can J Gastroenterol 20: 157-163.

22. Steigen SE, Eide TJ (2009) Gastrointestinal stromal tumors (GIST): a review. APMIS 117: 73-86.

23. Wang Yuan MENG Gang (2017) Clinicopathologic features of metastatic gastrointestinal stromal tumors. Chinese Journal of clinical and experimental pathology 33: 982-986.

24. LI Jun, et al. (2018) c-Kit and PDGFRA gene mutation in gastrointestinal stromal tumor and their associations with clinical pathological features, immunohistochemical expression and prognosis. J Clin Exp Pathol 34: 834-839.

25. Chou FF, Eng HL, Sheen-Chen SM (1996) Smooth muscle tumors of the gastrontestinal tract: analysis of prognostic factors. Surgery 119: 171-177.

26. Miettinen M, Killian JK, Wang ZF, Lasota J, Lau C, et al. (2013) Immunohistochemical Loss of Succinate Dehydrogenase Subunit A (SDHA) in Gastrointestinal Stromal Tumors (GIST) Signals SDHA Germline Mutation [J]. Am J Surg Pathol 37: 234-240.

27. Baskin Y, Kocal GC, Kucukzeybek BB, Akbarpour M, Kayacik N, et al. (2016) PDGFRA and KIT mutation status and its association with clinicopathological properties, including DOG1. Oncol Res 24: 41-53. 